\title{
Review of the Etiological Causes and Diagnosis of Myelitis and Its Medical Orientation Protocol
}

Besteiro B* and Guimarães J

Al. Prof. Hernâni Monteiro, 4200 - 319 Porto, Portugal

\begin{abstract}
Transverse myelitis (TM) is an inflammatory condition caused by a range of possibilities which can lead to a deregulation of our motor, sensory and autonomic systems. Therefore, it creates a major impact on personal and professional life. Because it causes great social and professional disability, it is crucial that the clinicians quickly recognize this condition so that treatment can be started thereby minimizing these negative effects. Thus, the aim of this review is to provide a practical clinical approach to the most important causal diseases of TM, clinical presentation and to elaborate a diagnostic algorithm that helps classifying and simplifying the work of the clinicians when facing a TM episode.
\end{abstract}

Keywords: Transverse myelitis; Multiple sclerosis; Neuromyelitis optica; outcome; MRI

\section{Introduction}

Transverse myelitis covers heterogeneous inflammatory conditions of the spinal cord which can have devastating neurologic effects such as motor, sensory, and autonomic dysfunctions [1].

It is imperative to note that myelopathy does not have the same meaning as myelitis, despite both terms imply spinal cord compromise due to a pathological event. On one hand, myelopathy can be caused by a range of etiologies. On the other hand, myelitis is referred to any spinal cord inflammatory event [2] awaiting further classification after the identification of the cause [3]. Another key reminder is that $\mathrm{TM}$ is currently used as a synonym to acute transverse myelitis in the literature [2].

For many years, an exact definition of TM or acute transverse myelitis (ATM) has been tried to be settled. The term "acute" varies depending on authors: Berman et al defined as more than $48 \mathrm{~h}$, Tranverse Myelitis Consortium Working Group between $4 \mathrm{~h}$ and 21 days following the onset of symptoms and Jeffery et al. as maximal aggravation in $24 \mathrm{~h} \mathrm{[3]}$

Besides this delineation, the term of TM is applied to any cause of inflammatory event, no matter what severity or degree of structural or functional interruption of pathways through a transverse spinal cord section [4].

Another well-established clarification of ATM is a bilateral medullar syndrome (symmetric or not) affecting motor, sensory and autonomic systems or, a lesion with $>50 \%$ of extension in MRI in a transversal plan [3].

It has become clear that TM patients have a heterogeneous display of injury, which can involve only gray matter, only white matter or both [1].

In the USA, 4,6 per million per year experience a TM episode. A study made by Young et al. demonstrated a bimodal distribution with two distinct peaks: 10-19 and 30-39 years old [5]. Racial/familial or gender predilection did not exist despite pediatric population has been affected in $28 \%$ of cases [5].

TM may be a severe condition once it could lead to poor outcome and have a major impact on professional life in one-third of patients. Because of this fact, it is important to have a long-term outcome [6]. $\mathrm{TM}$ can be divided into two subgroups based on the inflammation extent in the spinal cord: acute complete transverse myelitis (ACTM) and acute partial transverse myelitis (APTM) [7].

In ACTM, areas below to the lesion level are affected with a symmetric moderate to severe loss of the function. On the other hand, APTM affects at least one portion of the cross-sectional area of the spinal cord causing mild to severe weakness and sensory symptoms (asymmetric or dissociated) [7].

The role of MRI in accessing ATM has gotten more notorious since neuromyelitis optica (NMO) has been individualized. This imagiologic exam has enabled clarification of both diagnostic and prognostic values of longitudinally extensive transverse myelitis (LETM) [3] which typically shows intramedullary MRI lesion spreading throughout $\geq$ three juxtaposed segments [8] in the sagittal plane [3]).

Acute or subacute myelopathy are the typical clinical presentations of TM and, for no clear reasons, the thoracic cord is the most segment affected [5]. In acute myelopathy the progression to nadir of clinical deficits is between $4 \mathrm{~h}$ and 21 days following the onset of symptoms [9].

TM may arise due to different etiologies. It may be idiopathic when adequate diagnostic workup fails to reveal the causative factor of the lesion or secondary to other groups of diseases such as idiopathic demyelinating disease (multiple Sclerosis (MS), neuromyelitis optica (NMO) [5] or acute disseminated encephalomyelitis (ADEM) [9]) infectious causes (bacterial, viral, parasitic and fungal), secondary inflammatory diseases (paraneoplastic syndrome and systemic diseases such as systemic lupus erythematosus - SLE [5]) and also post vaccination [4]. Other causes such as metabolic [8] and vascular can also occur that can mimic TM [2].

In such causes, some have a progressive or relapsing course (such

*Corresponding author: Besteiro B, Al. Prof. Hernâni Monteiro, 4200 - 319 Porto, Portugal, Tel: 225-092-267; E-mail: bdds_b11@hotmail.com

Received January 31, 2017; Accepted February 24, 2017; Published February 28, 2017

Citation: Besteiro B, Guimarães J (2017) Review of the Etiological Causes and Diagnosis of Myelitis and Its Medical Orientation Protocol. J Neurol Neurophysiol 8: 415. doi:10.4172/2155-9562.1000415

Copyright: @ 2017 Besteiro B, et al. This is an open-access article distributed under the terms of the Creative Commons Attribution License, which permits unrestricted use, distribution, and reproduction in any medium, provided the original author and source are credited. 
Citation: Besteiro B, Guimarães J (2017) Review of the Etiological Causes and Diagnosis of Myelitis and Its Medical Orientation Protocol. J Neurol Neurophysiol 8: 415. doi:10.4172/2155-9562.1000415

as MS). On the other hand, some other causes can be monophasic with low risk for recurrence (like postinfectious and post vaccination [4]). For instance, cases have been described after vaccination against H1N1influenza [10].

Comparing the idiopathic form with secondary causes of TM, while the idiopathic form is a rare condition, secondary causes (such as MS) have a much higher prevalence being a common cause of TM [4]. Therefore, given the wide range of possibilities that can cause TM, it is crucial that clinicians are familiarized with this disease and they should be able to recognize TM clinical presentation, so that diagnosis and treatment can follow [4]. So, it is very important that the clinicians know how to recognize and diagnose myelopathies, the acute therapy to eliminate the inflammation, finding out the main cause and finally long term management [4].

The aim of this review is to discuss a practical clinical approach to the most important causal diseases of TM, clinical presentation and to create an algorithm diagnosis that helps classifying and simplifying the work of the clinicians in the differential diagnosis of patients with TM.

Although several excellent reviews on this topic are available, this review adds value once it summarizes a vast literature for the diagnosis of TM and includes some of the most important articles published from the earliest to recent data. The suggested medical orientation protocol is very practical and useful to clinicians addressing any etiology and it might be helpful to them when facing a case of suspected TM, requiring a rapid approach (Chart 1$)$.

\section{Methods}

For this review, an expert opinion based on retrospective studies of myelitis search on PubMed was the base to write this review (Table 1). Scopus and ISI Web of Science database were consulted through the

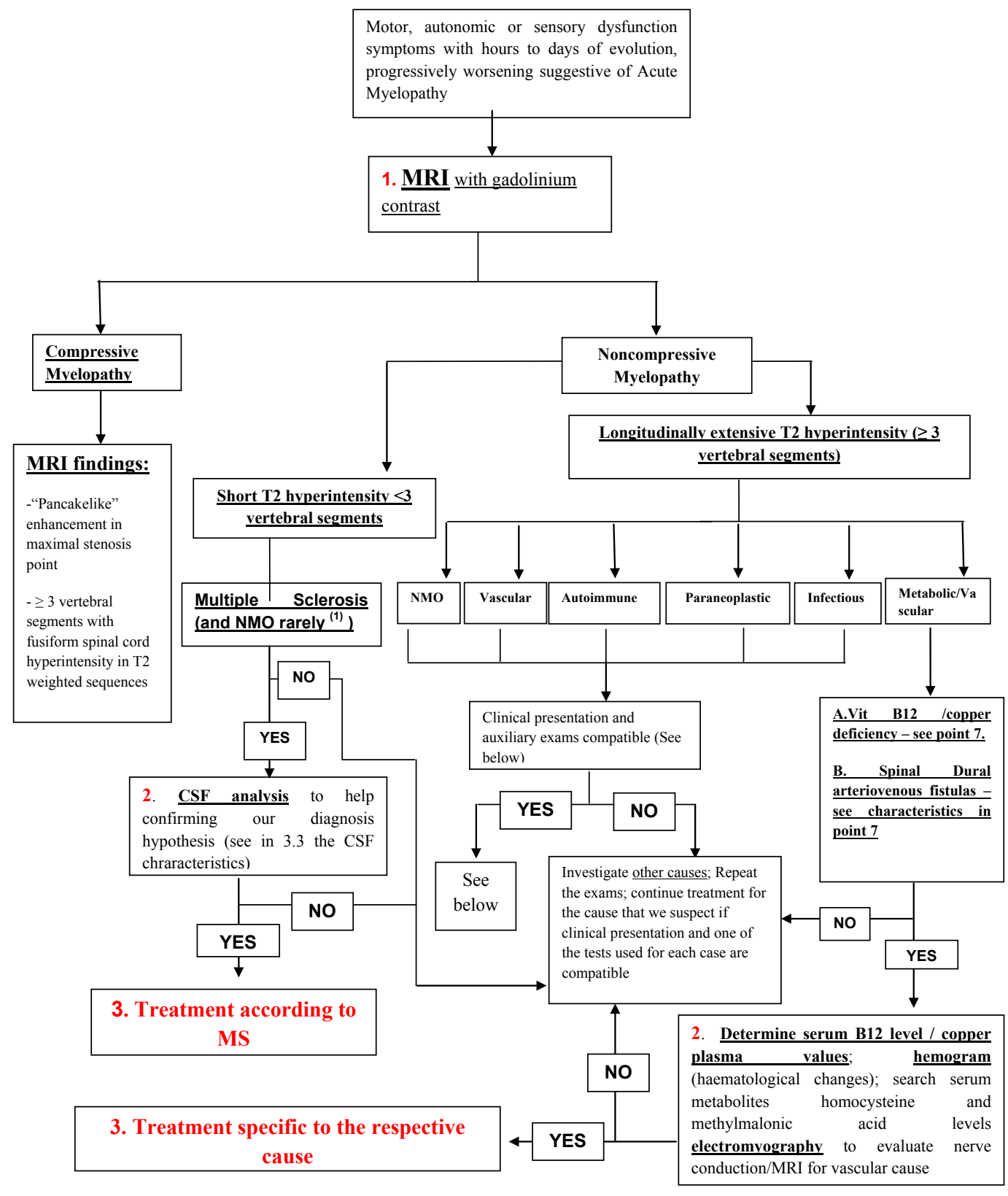




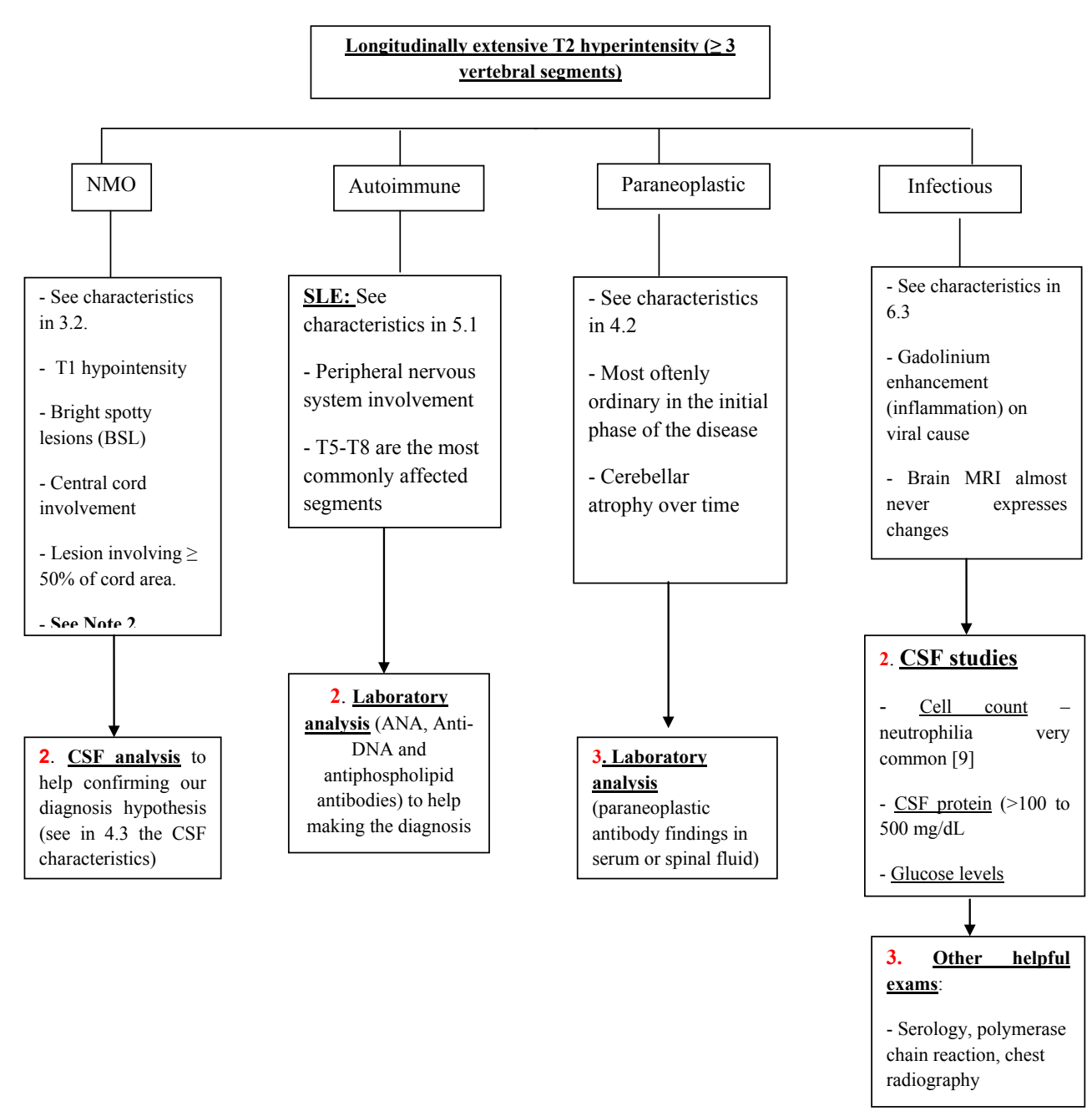

Chart 1: Medical orientation protocol.

(1) In addition, a small portion of patients with NMO can also have Short T2 hyperintensity lesions

Note 1: If MRI is normal, the clinician should search or alternative explanations consistent with a normal MRI. If there are no explanations, repeat MRI and CSF analysis could be possible alternatives

Note 2: In a study performed, it was found that in NMO spectrum disorders, short TM (<3 vertebral segments) is not uncommon (14\% occurred as a first event in TM vs LETM-which occurred in $86 \%$ ) and it may delay the diagnosis and treatment of this disease when it is present. Short myelitis lesions in MRI do not exclude consideration of AQP-4-IgG testing! [11].

central library of the Faculty of Medicine of Porto University. We searched articles published in English, Spanish and French and included the MeSH terms transverse myelitis, multiple sclerosis, neuromyelitis optica and MRI.

Sixty of the one hundred retrieved articles were excluded, once only articles related to at least 2 mesh terms (including transverse myelitis the main theme of this work) were included. Many articles which were excluded were not related to the main theme and focused on other issues that were not relevant to this article. 2016.

The closing date of the literature search was from 1993 to December

After reading each article we analyzed the conclusions of each one in order to select the posterior articles (which were review articles with the same MeSH terms with the exception of retrospective studies).

In every search made, only items with links to full text, and a publication date no further back than 1993 were selected. Other articles referred in this review that were not produced in the original search, were located in data bases through other articles' own references or were specifically suggested by a specialist in the area. In total, 47 articles are referred in this expert opinion.

\section{TM and Idiopathic Demyelinating Disease \\ TM and multiple sclerosis (MS)}

Epidemiology: MS is an auto-immune demyelinating disease of the central nervous system (CNS) characterized by chronic inflammation and demyelination of unknown etiology. MS may present with acute myelitis, particularly in Caucasians, Northern European ancestry individuals and female sex individuals (who are diagnosed 2 to 3 times more that of men) [19].

Its prevalence ranges from 2 per 100,000 in Japan to greater than 100 per 100,000 in Northern Europe and North America. MS is influenced 
Citation: Besteiro B, Guimarães J (2017) Review of the Etiological Causes and Diagnosis of Myelitis and Its Medical Orientation Protocol. J Neurol Neurophysiol 8: 415. doi:10.4172/2155-9562.1000415

Page 4 of 8

\begin{tabular}{|c|c|c|c|}
\hline $\begin{array}{l}\text { STUDYI } \\
\text { AUTHOR'S }\end{array}$ & Number of patients & Classification & Major results \\
\hline Douglas et al. [12] & $\begin{array}{l}33(45 \% \text { categorized as } \\
\text { parainfectious and } 21 \% \text { as } \\
\text { MS) }\end{array}$ & $\begin{array}{l}\text { Cases were classified as } \\
\text { being related to parainfectious } \\
\text { MS, spinal cord ischemia or } \\
\text { idiopathic. }\end{array}$ & $\begin{array}{c}\text { Patients with parainfectious TM showed evidence of spinal cord swelling, whereas } \\
\text { patients with MS-associated TM had spinal cord plaques on MRI but none } \\
\text { showed swelling. Oligoclonal bands were absent in patients with parainfectious } \\
\text { TM and present in three of five patients with MS- associated TM. }\end{array}$ \\
\hline \multirow[b]{3}{*}{ Harzheim et al. [13) } & \multirow[b]{3}{*}{$\begin{array}{c}45 \text { (Parainfectious (38\%); } \\
\text { Idiopathic (36\%); MS ( } 22 \%) ; \\
\text { Rheumatoid arthritis }(2 \%) ; \\
\text { Hypersensitivity vasculites } \\
(2 \%)\end{array}$} & \multirow{3}{*}{$\begin{array}{c}45 \text { unselected consecutive } \\
\text { ATM patients diagnosed at } \\
\text { the Department of Neurology } \\
\text { in Germany were classified } \\
\text { for this study }\end{array}$} & 1. Clinical findings: Motor weakness (49\%) and Tetraparesis $(11 \%)$ \\
\hline & & & $\begin{array}{l}\text { 2. Laboratory and CSF findings : Blood tests abnormal (66\%) and CSF findings } \\
\text { abnormal in } 84 \%\end{array}$ \\
\hline & & & $\begin{array}{l}\text { 3. MRI findings : (1) signal alterations on T2-weighted images }(96 \%) ;(2) \text { single } \\
\text { lesions restricted to one spinal segment }(78 \%) ;(3) \text { Of a total number of } 54 \text { spinal } \\
\text { cord lesions identified, } 34 \text { were located in the cervical cord, } 14 \text { located at thoracic } \\
\text { level and, } 4 \text { between cervical-thoracic junction; (4) Cranial MU was performed in } \\
34 / 45 \text { and half of these patients, multiple signal alterations were found . with more } \\
\text { than } 50 \% \text { of these last abnormalities indicating MS presence. }\end{array}$ \\
\hline \multirow{4}{*}{ Pidcock et al. [14] } & \multirow{4}{*}{47} & \multirow{4}{*}{$\begin{array}{l}47 \text { cases with prior ATM } \\
\text { occurred under the age of } \\
18 \text { years where classified for } \\
\text { this study. }\end{array}$} & $\begin{array}{l}\text { 1. Demographic features and risk factors demonstrated two possible peaks of } \\
\text { incidence (c3 years and between } 5 \text { and } 17 \text { years); No predominance gender }\end{array}$ \\
\hline & & & $\begin{array}{l}\text { 2. Clinical Findings : Mean time from the onset of acute symptoms to functional } \\
\text { nadir was } 2 \text { days; } 91 \% \text { had a sensory loss and } 89 \% \text { weakness , } 85 \% \text { urinary } \\
\text { disfunction }\end{array}$ \\
\hline & & & $\begin{array}{l}\text { 3. MR1 findings: (1) In } 38 \text { patients who made it, half of them demonstrated T2 } \\
\text { signal abnormalities located in the cervical cord and lesions located at thoracic } \\
\text { level in } 40 \% \text { of then (2) In } 38 \% \text { of patients, a hypointense lesion was revealed: } \\
\text { (3) T1-weighted gadolinium-enhancing lesion in } 74 \% \text { of cases. }\end{array}$ \\
\hline & & & $\begin{array}{l}\text { 4. CSF findings CSF was elevated ( white blood count with a mean of } 136+-67 \\
\text { cells and } 48 \% \text { of patients with CSF protein level }\end{array}$ \\
\hline Chaves et al. [15] & 40 & $\begin{array}{l}40 \text { patients diagnosed with } \\
\text { ATM between June } 1,2002 \\
\text { and June } 30,2010 \text { was } \\
\text { retrospectively identified } \\
\text { based on the Transverse } \\
\text { Myelitis Consortium Working } \\
\text { Group (TMCWG) criteria. }\end{array}$ & $\begin{array}{l}\text { - In } 40 \text { patients ( } 60 \% \text { female) with ATM, demyelinating disease was the most type } \\
\text { of this disease; Idiopathic transverse myelitis was the cause in } 37,5 \% \text { of cases } \\
\text { - Most of the cases presented as extensive longitudinally transverse myelitis in } \\
\text { MRI }\end{array}$ \\
\hline Calvo et al. [16] & $\begin{array}{l}87 \text { (Eleven (13\%) patients } \\
\text { converted to MS After a } 2.9 \\
\text { years of follow-up) }\end{array}$ & $\begin{array}{c}87 \text { patients diagnosed with } \\
\text { Idiopathic ATM between } 1989 \\
\text { and } 2011 \text { were retrospectively } \\
\text { reviewed. }\end{array}$ & $\begin{array}{l}\text { - MS conversion is related with a more early age of onset } \\
\text { - Presence of LETM on MRI at admission as well as urinary sphincter dysfunction } \\
\text { were associated to a worse outcome }\end{array}$ \\
\hline & $64(32.81 \%$ were clinically & $\begin{array}{l}64 \text { patients diagnosed with } \\
\text { LETM }\end{array}$ & $\begin{array}{l}\text { 1. Clinical findings: the majority of the patients presented with acute bladder } \\
\text { dysfunction and paraparesis. }\end{array}$ \\
\hline Jain et al. [17] & $\begin{array}{c}\text { diagnosed as NMO; } \\
\text { Others with MS, ADEM, } \\
\text { postinfectious, tuberculous } \\
\text { myelitis, spinal arteriovenous } \\
\text { malformation, SLE and } \\
\text { idiopathic) }\end{array}$ & $\begin{array}{l}\text { were retrospectively reviewed } \\
\text { from August } 2010 \text { to February } \\
2016 \text { and demographic } \\
\text { profile, clinical findings, } \\
\text { laboratory parameters and } \\
\text { etiological causes were } \\
\text { analyzed }\end{array}$ & $\begin{array}{l}\text { 2. MRI findings: Brain and Spine MRI were normal in } 70 \% \text { of patients and } \\
\text { abnormal in } 31.25 \% \text { of them In spine MRI, in } 64.06 \% \text { of patients } 3-6 \text { segments } \\
\text { were involved and more than } 6 \text { segments were involved in } 35.93 \% \text { of them }\end{array}$ \\
\hline Rodriguez et al. [18] & 91 & $\begin{array}{l}91 \text { patients diagnosed } \\
\text { with myelitis episode were } \\
\text { retrospectively reviewed from } \\
2000 \text { to } 2013 \text {. Demographic } \\
\text { profile, etiological causes, } \\
\text { clinical, radiological and } \\
\text { prognostic variables were } \\
\text { analysed and compared } \\
\text { between patients with myelitis } \\
\text { caused by MS and those } \\
\text { with myelitis due to other } \\
\text { etiologies. }\end{array}$ & $\begin{array}{l}\text { 63\% were diagnosed with MS with earlier ages of onset, more prominent } \\
\text { sphincter impairment and greater multifocal involvement in spinal MRI. } \\
\text { Cervical and posterior location was more related to Myelitis due to MS.E19 } \\
\text { Other etiologies differed in the location (more often anteriorly) and central cord } \\
\text { involvement showed better recovery at one year of follow up }\end{array}$ \\
\hline
\end{tabular}

Table 1: Major retrospective studies of acute myelitis.

by longevity and comorbidities [20] such as cigarette smoking and Vitamin D levels $<75 \mathrm{nmol} / \mathrm{L}[19]$.

Its impact on health economy is relevant in many countries [21].

Clinical presentation: When TM is firstly diagnosed, MS is one of the main etiologies of TM syndromes [7].

It is known that patients with APTM possibly have a higher risk to develop MS when compared to patients presenting ACTM [7]. A study also revealed that in $44-93 \%$ of patients who experience a partial TM episode had a conversion to MS [5].
Characteristically, when TM occurs in MS patients the lesion is very often located dorsally reaching less than three [21] or two [9] vertebral segments (in other words, is a short segmented lesion) [4]. The lesions are usually small and peripheral causing asymmetric symptoms and signs [9].

There are some typical clinical MS presentations associated with TM. Lhermitte's sign - intense burst of pain like an electric shock that occurs in neck flexion - is a characteristic presentation [22]. BrownSéquard syndrome may also be a clinical presentation [9].

The clinical presentation of MS based on a review which compared 
clinical presentations among different TM etiologies, showed the following occurrences in 7 patients with MS: positive/negative symptoms ( $57 \%$ with both), sensory level (cervical - $43 \%$, thoracic $43 \%$ and lumbar - 14\%), mostly asymmetric lesions occurred (86\%), level progression (43\% ascending, $0 \%$ descending and $57 \%$ none), sensory dissociation (mostly did not show $-67 \%$ ), deep tendon reflex normoactive (60\%) or hyperactive (40\%) [1].

Auxiliary diagnostic exams: When TM is associated with MS, auxiliary diagnostic exams such as spinal cord MRI, brain MRI and cerebrospinal fluid (CSF) analysis are required [9].

MS often discloses diagnostic clues that help clinicians: spinal cord MRI usually reveals lesions which broaden less than 2 vertebral segments, preferably in lateral and posterior funiculi, normally peripherally located [9]. In brain MRI, white matter lesions and Dawson's fingers are characteristic. Lesions may be located in periventricular, juxtacortical or infratentorial zone [9].

Finally, CSF analysis shows the presence of oligoclonal bands (OCB) in more than $90 \%$ of patients and increased IgG index in more than $60 \%$ of patients [9]. CSF analysis for OCB is advantageous in that determining MS vs other causes of TM such as NMO, spinal cord infarct, vasculitis parainfectious and idiopathic TM [7].

The CSF can add useful information about inflammatory and immunological alterations in patients with clinical presentation or radiological findings similar to MS and allow the exclusion of MS mimickers (namely infectious and neoplastic conditions) [7]. CSF pleocytosis is rare $(\leq 50$ cells $/ \mu \mathrm{L})$ and CSF protein is mildly elevated $<100 \mathrm{mg} / \mathrm{dL}$ ) [23].

It is known that $85 \%$ of MS patients will present a TM episode during the course of their disease. On the other hand, TM can also be the initial presentation in approximately 20 to $40 \%$ in MS patients [4].

When MS patients present a TM event, a short segmented lesion, partial pattern on MRI, positive oligoclonal band testing in CSF and a brain MRI presenting $\geq$ two lesions consistent with demyelination lesion, are signs that predict MS [4].

According to a review, after an initial APTM episode and the presence of $\geq$ two lesions, there is $88 \%$ probability of MS conversion in the next 20 years [9]. However, it is important to note that none of these four lesions aforementioned are $100 \%$ predictive of MS and therefore many other possibilities must be considered [4].

If instead all the parameters mentioned above are normal, the probability of development MS would be as low as 10\%-30\% [4] or only $19 \%$ for those whose MRI are free of abnormalities [9].

A study published in 2012 that aimed to evaluate the risk of conversion from APTM to MS (after 8.7 years of follow-up) confirmed that white matter lesion on initial brain MRI and the presence of OCBs in CSF are two risks factors for this conversion to occur, with an odds ratio of 7.7 (95\% CI 2.42-24.74) and 15.8 (95\% CI, 2.95-84.24) respectively. However, clinical, biological and radiological findings did not predict a long-term disability in MS patients [24].

\section{TM and neuromyelitis optica (NMO)}

Epidemiology: Neuromyelitis optica is an idiopathic, aggressive, inflammatory and demyelinating disorder [5] characterized by recurrent attacks in CNS affecting predominantly the optic nerve (ON) - optic neuritis and the spinal cord - myelitis [9]. This condition is more frequent in women than in men, in a 3:1 ratio [2].The onset occurs mostly during adulthood with a median age in the late 30's [5]. However, it may also begin sooner, in pediatric time, with an average age of 10 years old [5].

Clinical presentation: NMO as the main cause of TM presents some clinical findings which may help the clinicians [2].

The presence of optic neuritis and acute myelitis is very characteristic [2]. The ultimate criteria for adult patients are well defined and they can be followed depending on the presence/absence of clinical markers and clinical presentation (Table 1).

The attacks on can be unilateral (more common) or bilateral, and a history of previous optic neuritis should raise suspicion of NMO [9]. Optic neuritis and acute myelitis usually occur sequentially rather than simultaneously [24]. Therefore, not only previous optic neuritis but also myelitis should create awareness towards NMO [9].

A study performed in 2008, with most of the NMO patients being women, revealed that the onset was acute in about one-third of them. Motor deficits and notorious functional impairment were found in $90 \%$ of the cases [6].

The clinical presentation of NMO based on the same review reported above, showed the following occurrences in 9 patients with NMO: positive/negative symptoms (56\% with both), sensory level (cervical $-11 \%$, thoracic - $89 \%$ and lumbar - $0 \%$ ), mostly asymmetric lesions occurred (67\%), level progression (33\% ascending, $45 \%$ descending and $22 \%$ none), sensory dissociation (100\%), deep tendon reflex normoactive (80\%) or hyperactive (20\%) [1] (Table 2).

\section{Patient's findings}

A) NMO spectrum disorders with AQP-4 IgG

B) NMO spectrum disorders without AQP-4 IgG or NMO Spectrum disorders with unknown AQP-4 IgG status

Further MRI demands for NMO spectrum disorders without AQP-4 $\lg$ and NMO spectrum disorders with unknown AQP-4 IgG status

Core clinical characteristics

\section{Diagnostic criteria}

$\geq 1$ core clinical characteristic (see below on Core clinical characteristics) AND positivity for AQP-4 IgG (using the best detection method) AND exclusion of other differential diagnosis [8]

$\geq 2$ clinical findings occurred due $\geq 1$ clinical attacks

Presenting all of the topics :

21clinical disease: Optic Neuritis, acute myelitis with LETM lesions or area postrema syndrome, dissemination in space and achievement of further MRI requirements),

Negativity for AQP-4 IgG (using the best detection method) or testing unavailable and exclusion of other differential diagnosis [8]

Acute optic neuritis needs: Brain MRI displaying non-pathological findings or exclusively nonspecific white matter lesions $\underline{\text { OR }}$ Optic nerve MRI with T2-hyperintense lesions or T1-weighted gadolinium-enhancing lesion branching over 9 one-half optic nerve length or compromising optic chiasm)

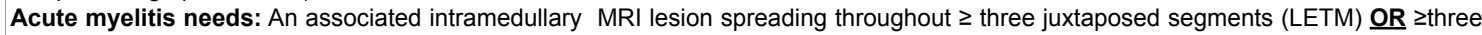
juxtaposed segments of focal spinal cord atrophy in patients with previous history consistent with acute myelitis),

Area postrema syndrome: with concomitant lesion in dorsal medulla/area postrema

Acute brainstem syndrome: with concomitant lesions in periependymal brainstem [8].

Optic Neuritis; Acute myelitis; Area postrema syndrome; Acute brainstem syndrome

Symptomatic narcolepsy or acute diencephalic clinical syndrome with NMO spectrum disorder and symptomatic cerebral syndrome with NMO spectrum disorder [8].

Table 2: NMO Spectrum disorder diagnostic criteria for adult patients. 
Auxiliary diagnostic exams: After clinical presentation, subsidiary exams are required to confirm/exclude the presence of NMO.

Several features of TM are associated with NMO, including a LETM extending over 3 or more vertebral segments [4], confluent lesions [2], a centrally located lesion within the cord, extensive cord swelling [4] or absence of white matter lesions [2]. The spinal cord lesion length is an extremely important clue to help in the differential diagnosis between NMO and MS (which typically reaches less than three or two vertebral segments) [25].

Additional clues include brainstem lesions which can occur isolated or as a rostral extension of cervical myelitis [2]. This area's involvement may provoke nausea and vomiting due to compromise of the area postrema in the lower medulla [4].

A MRI is very helpful in diagnosing NMO [5]. The spinal cord MRI typically discloses lesions extending over three or more spinal cord segments (LETM), cord swelling and gadolinium enhancement in acute lesions [9].

Common imagiologic findings on MRI are T1 hypointensivity [8], bright spotty lesions [26], central cord involvement [8] and lesion involving $\geq 50 \%$ of cord area [26]. In brain MRI, lesions are present in up to $60 \%$ of patients, usually in periventricular zone [9].

CSF findings include greater pleocytosis than that observed in MS, with neutrophils and eosinophils present in some cases during acute attacks. OCB are uncommon and are not present in more than $80 \%$ of cases [9].

Although these clinical features are the most recurrent in NMO presentation, clinicians should be conscious that NMO might lead to a variety of TM patterns like short segment TM (instead of long extensive pattern) or even not cause any of these sorts [4].

As a result of this very reason, all patients should be tested for AQP4 antibodies which help determining the cause of TM. Because of the variety of patterns which can occur in $\mathrm{NMO}$, it is reasonable to consider that all patients with TM should be tested to AQP-4 antibodies at least once (although it is not feasible in routine practice) [4].

These autoantibodies are highly specific $(>90 \%)$ and sensible (>70\%) for NMO [9] and a positive result should prompt clinicians to treat patients with NMO [4]. However, in $10-30 \%$ of patients with clinical NMO, the AQP-4 antibodies are not present. Despite this, they should be treated like patients with positive AQP-4 antibodies [4].

Concerning prognosis, NMO is associated with a worse functional outcome of an acute or subacute myelopathy (ASM) episode. The disease is also related to a worse professional activity with a major impact [6].

In a study performed, it was found that when ASM occurred, it often consisted not only a unique event but also a neurologic event occurred in almost half of the patients. In addition, the AM event leads to chronic conditions such as MS, NMO and systemic disease in more than half of the cases which were initially diagnosed as myelopathy of undetermined cause [6] (Table 3).

\section{TM and Paraneoplastic Disorder}

\section{Epidemiology, pathophysiology and clinical presentation}

Despite being rare, diagnosing paraneoplastic disorder is essential once diffuse CNS abnormalities are the most frequent setting in which this cause occurs [4].

Collapsin response mediator protein-5 (CRMP-5) and antiamphiphysin are two antineuronal antibodies which are very often associated with paraneoplastic syndrome. Their detection raises the possibility of lung and breast cancer [4]. There are other cancers associated with paraneoplastic disorders as well as their antibodies like breast cancer (PCA 2), ovarian cancer (ANNA 2), non-small cell lung cancer (neuronal and muscle AChR antibodies) [9].

Clinically, an insidious presentation is very common in paraneoplastic myelopathies oppositely to MS and NMO which typically have a more acute onset [4].

\section{Auxiliary diagnostic exams}

In addition to the antibodies, MRI is also required and it often shows tract-specific hyperintense signal changes, such as in the corticospinal tract [4]. In addition, Positron-emission tomography (PET) scan may increase the diagnostic yield for certain cancers where other modalities have been negative/uninformative. PET imaging alone, or in combination with anatomic data (PET-CT), increases the cancer diagnostic yield by $20 \%$ [31].

It should also be noted that lymphocytic pleocytosis and oligoclonal

\begin{tabular}{|c|c|c|}
\hline Findings/Disease & Multiple Sclerosis & NMO \\
\hline Brain MRI & $\begin{array}{l}\text { 1: White matter lesions [9]; } \\
\text { 2: Dawson's fingers [9]; } \\
\text { 3: Periventricular, juxtacortical, } \\
\text { infratentorial lesions [9]. }\end{array}$ & $\begin{array}{l}\text { 1: Lesions present in up to } 60 \% \text { of patients [9]; 2: Lesions located periventricular zone and } \\
\text { brainstem a (involving more frequently its central and dorsal aspects [28]). 3: Lesions localized } \\
\text { at sites of high AQP4 expression such as in hypothalamus }{ }^{a}[28] \cdot 4: \text { Corticospinal tract lesions } \\
\text { frequently bilateral involving the posterior limb of the internal capsule and the cerebral peduncle } \\
\text { [28]. 5: Lesions lining the ependymal surface of the lateral ventricles }{ }^{\text {a }}[28] .6 \text { : Acute phase - } \\
\text { large edematous and heterogeneous lesions in the corpus callosum; Chronic phase - reduction } \\
\text { in their size and intensity or even its disappearance }{ }^{a}[28] \text {. }\end{array}$ \\
\hline Spinal Cord MRI & $\begin{array}{l}\text { 1: Less than three [19] or two vertebral } \\
\text { segments usually peripherally located [9]; } \\
\text { 2: Lateral or posterior funiculi preferred [9]. }\end{array}$ & $\begin{array}{l}\text { 1: >three vertebral segments (or short lesions - see note } 2 \text { at section } 8 \text { ) [9]; 2: cord swelling [9] } \\
\text { and a gadolinium enhancement (cloud-like lesions }{ }^{\text {a }} \text { [28]) varying between } 31,2 \%[29] \text { to } 85 \% \text { in } \\
\text { acute lesions [30]; 3: T1 hypointensivity [8]. }\end{array}$ \\
\hline CSF-OCB and others & $\begin{array}{l}\text { 1: Present and raised IgG index [9]. OCB } \\
\text { present in } 90 \% \text { of cases [8]. } \\
\text { 2: CSF glial fibrillary acidic protein (CSF- } \\
\text { GFAP) during relapse is normal or mildly } \\
\text { elevated [8]. }\end{array}$ & $\begin{array}{l}\text { 1: OCBs are often absent }(\leq 25 \%)[8] \text {. AQP-4 lgG usually presents [8]. } \\
\text { 2: CSF - GFAP usually very elevated [8]. }\end{array}$ \\
\hline CSF - Pleocytosis & $\begin{array}{l}\text { 1: The White blood cell count (WBC) } \\
\text { is usually }<50 \text { cells } / \mathrm{mm}^{3} ; \text { Lymphocytic } \\
\text { predominance [5]; Mild pleocytosis [8]. }\end{array}$ & $\begin{array}{l}\text { 1: WBC is usually } 0-50 \text { cells } / \mathrm{mm}^{3} \text {; neutrophils and eosinophils present in some cases [5]; 2: } \\
\text { Pleocytosis more prominent during the acute attack of TM rather than ON [5]; 3: Pleocytosis has } \\
\text { a great specificity }(95 \%) \text { despite having a sensibility }<30 \%[5] \text {. }\end{array}$ \\
\hline $\begin{array}{c}\text { Secondary Progressive } \\
\text { course }\end{array}$ & Common [27]. & Rare [27]. \\
\hline
\end{tabular}

Table 3: Differential diagnosis between MS and NMO in myelitis presentation (a-According to a study these are the most distinctive brain lesions found on MRI in NMO). 
bands are present in $30 \%$ of patients and these results might complicate the diagnosis to the clinician [4].

\section{TM and Other Autoimmune Diseases (Secondary Inflammatory Diseases)}

\section{Epidemiology, clinical presentation and auxiliary diagnostic} exams

Systemic lupus erythematosus (SLE) and Sjögren syndrome are two major autoimmune diseases associated with TM [2].

It is estimated that 1-3\% of patients with SLE present myelopathy [32]. Other article states that TM as a manifestation of SLE has a 1-2\% prevalence in the same patients [33]. Women are very often affected when compared with men in an 8:1 ratio [2].

In the majority of the cases, acute episodes of TM are the initial symptom as well as an earlier symptom present in the early course of the disease [34]. In the majority of the cases, ATM develops over several hours and worsens within the next several days. Less frequently, patients will worsen over the course of several weeks [35]. Although it is still more common for SLE symptoms to precede those of TM, it is known that $23 \%$ to $39 \%$ of SLE patients have TM symptoms as their initial presentation [35].

During diagnosis, it is appropriate to start with with serologies for NMO-IgG/AQP4 antibodies, CSF analysis, and blood testing [35]. The types of achievements found in both diseases are similar to those found in NMO. Myelitis occurs in a small number of patients with SLE, many cases of which are associated with antibodies against antiphospholipids and/or against aquaporin-4. Therefore, patients with aquaporin-4 antibodies are likely to display LETM in MRI [4], ranging more than 4-segments [32] and T5-T8 are the most commonly affected segments [34].

It is known that a majority of patients with NMO have serologic markers of SLE or Sjögren syndrome without having systemic symptoms of these diseases [4].

The CSF in SLE myelitis is usually normal or shows a mild lymphocytic pleocytosis; oligoclonal bands are a variable finding but they are generally rare [2].

\section{TM and Infectious Diseases}

\section{Causal agents}

Many agents can be a cause of infectious myelitis. Among these agents viruses, bacteria, fungal and parasites are the most important [9].

Looking deeper into viruses as a cause, many of them have been associated with acute myelitis such as DNA viruses and RNA viruses [9]. Among DNA viruses, Herpes Simplex virus-1 (HSV-1) [36], Herpes simplex vírus-2 (HSV-2), Varicella-Zoster vírus (VZV), Cytomegalovirus (CMV), Human Herpes viruses 6 and 7 (HHV 6 and 7) and Epstein-Barr virus (EBV) are noteworthy [9]. In RNA viruses, it is important to remind Dengue virus, Influenza A virus, human T-lymphotropic virus (HTLV) and Hepatitis A and C virus [9].

Reporting the most relevant information for some of them, HSV1 can be the reason behind an unusual descending paralysis function [36]. HSV-2 produces Elsberg's syndrome [37], EBV can rarely cause $\mathrm{TM}$ and is associated with a poor prognosis [38]. Dengue viral infection could cause an ATM episode as a manifestation, despite only four cases having been reported in the literature [39]. HTLV causes a slow progressive myelopathy associated to a spastic paraparesis which is often difficult to diagnose as it can be masked by common entities [40].

There are other possible etiological agents such as Mycoplasm, Treponema pallidum, Mycobacterium tuberculosis, Brucella, Borrelia [2], Actinomyces, Blastomyces dermatitidis, Coccidioides and Apergillus [9].

Schistosoma [9] which is an important cause of TM in endemic areas [40], toxoplasma, cysticercus and echinococcus can also cause TM [9].

\section{Clinical presentation}

In the clinical presentation of infectious causes, fever and meningismus are frequently present and auxiliary exams are ordinarily required [9]. Many other clinical clues indicate an infectious cause such as confusion, rash, concurrent systemic infection, immunocompromised state, recurrent genital infection, lymphadenopathy and also the fact that the patient is living in endemic areas [9].

\section{Auxiliary diagnostic exams}

For elucidating the etiology of infectious myelitis CSF studies are essential. CSF analysis usually reveals pleocytosis, commonly neutrophilia [2], protein elevation [9] (higher than 100 to $500 \mathrm{mg} / \mathrm{dL}$ in children) and lower glucose levels [41].

Spinal and brain MRI are also required and while spinal MRI shows abnormalities as T2 hyperintensity, brain MRI almost never expresses changes (despite very similar changes to what happens in acute demyelinating encephalopathy having been demonstrated in white matter [2].

Other exams could be required to sort differential diagnosis to other TM etiologies as stains and cultures, CSF polymerase chain reaction (for viruses and Borrelia burgdorferi), serology, blood cultures and also chest radiograph/CT [9].

It should be noted that direct culture of Borrelia species and CSF PCR is known to have low sensibility [42]. Therefore, intrathecal production of immunoglobulins (assessed by antigen-specific oligoclonal IgG demonstration) and especially an intrathecal synthesis of Borrelia specific antibodies in CSF and serum are the best indicators for definitive diagnosis [43].

\section{TM and Metabolic and Vascular Disorders}

In the study of a spinal cord inflammatory event, it is fundamental to contemplate two disorders that must be considered and excluded: metabolic and vascular disorders.

Metabolic causes such as Vitamin B12/copper deficiency can cause AM episode and this vitamin deficiency can be a mimicker of the idiopathic demyelinating disease. The spinal cord manifestation is characterized typically by sensory symptoms that can mimic demyelinating diseases such as symmetric dysesthesia, disturbance of position sense and spastic paraparesis or tetraparesis [44].

In brain MRI, ventricular garlands, spinal cord generation [8] and longitudinal high T2 signal lesions in cervical and upper thoracic segments can be visualized [45].

In vascular disorders, it is important to exclude arteriovenous fistulas (AVFs) (which have a slower progression due to gradual ischemia) [4]. Symptoms of spinal dural AVFs may be mistaken for 
Citation: Besteiro B, Guimarães J (2017) Review of the Etiological Causes and Diagnosis of Myelitis and Its Medical Orientation Protocol. J Neurol Neurophysiol 8: 415. doi:10.4172/2155-9562.1000415

more common spinal demyelinating diseases such as multiple sclerosistransverse myelitis [46].

Despite improvements in spinal imaging, AVF diagnosis is often difficult or masked by more common entities. Magnetic resonance (MR) imaging typically shows dilated perimedullary veins [47] and central high signal intensities on T2-weighted image [8].

\section{References}

1. Hyun JW, Kim JY, Choi KG, Kim HJ, Park KD (2015) The etiological spectrum of acute sensory myelitis. J Clin Neurol 11: 227-233.

2. Sánchez AM, Posada LMG, Toscano CAO, López AL. (2011) Diagnostic approach to myelopathies. Rev Colomb Radiol 22: 1-21.

3. Collongues N, Papeix C, H Zéphir, Audoin B, Cotton F et al. Nosology and etiologies of acute longitudinally extensive transverse myelitis. Rev Neurol (Paris) 170: 6-12.

4. Greenberg BM, Frohman EM (2015) Immune-mediated myelopathies. Continuum (Minneap Minn 21: 121-31

5. Awad A, Stüve O (2011) Idiopathic transverse myelitis and neuromyelitis optica: Clinical profiles, pathophysiology and therapeutic choices. Curr Neuropharmacol 9: 417-428.

6. Debette S, de Sèze J, Pruvo JP, Zephir H, Pasquier F, et al. (2009) Long-term outcome of acute and subacute myelopathies. J Neurol 256: 980-988.

7. Scott TF, Frohman EM, De Seze J, Gronseth GS, Weinshenker BG (2011) Evidence-based guideline: clinical evaluation and treatment of transverse myelitis: Report of the therapeutics and technology assessment subcommittee of the American Academy of Neurology. Neurology 77: 2128-2134.

8. Sand IK (2016) Neuromyelitis optica spectrum disorders. Continuum (Minneap Minn) 22: 864-896.

9. Jacob A, Weinshenker BG (2008) An approach to the diagnosis of acute transverse myelitis. Semin Neurol 28: 105-120

10. Austin A, Tincani A, Kivity S, Arango MT, Shoenfeld Y (2015) Transverse myelitis activation post-H1N1 immunization: A case of adjuvant induction? Isr Med Assoc J 17: 120-122.

11. Flanagan EP, Weinshenker BG, Krecke KN, Lennon VA, Lucchinetti CF, et al (2015) Short myelitis lesions in aquaporin-4-lgG-positive neuromyelitis optica spectrum disorders. JAMA Neurol 72: 81-87.

12. Jeffery DR, Mandler RN, Davis LE (1993) Transverse myelitis. Retrospective analysis of 33 cases, with differentiation of cases Associated with multiple sclerosis and parainfectious events. Arch Neurol 50: 532-535.

13. Harzheim M, Schlegel U, Urbach H, Klockgether T, Schmidt S (2004) Discriminatory features of acute transverse myelitis: A retrospective analysis of 45 patients. J Neurol Sci 217: 217-223.

14. Pidcock FS, Krishnan C, Crawford TO, Salorio CF, Trovato M, et al. (2007) Acute transverse myelitis in childhood: center-based analysis of 47 cases. Neurology 68: 1474-1480.

15. Chaves M, Rojas JI, Patrucco L, Cristiano E (2012) Acute transverse myelitis in Buenos Aires, Argentina. A retrospective cohort study of 8 years follow-up. Neurologia 27: 348-353.

16. Calvo AC, Martínez MA, Alentorn-Palau A, Escuer JB, Pinel LR, et al. Idiopathic acute transverse myelitis: Outcome and conversion to multiple sclerosis in a large series. BMC Neurol 13: 135

17. RS Jain, Kumarb S, Mathur T, Tejwani S (2016) Longitudinally extensive transverse myelitis: A retrospective analysis of sixty-four patients at tertiary care center of north-west India. Clin Neurol Neurosurg 148: 5-12.

18. Presas-Rodríguez S, Grau-López L, Hervás-García JV, Massuet-Vilamajó A Ramo-Tello C (2016) Myelitis: Differences between multiple sclerosis and other aetiologies. Neurologia 31: 71-75.

19. Maroney M, Hunter SF (2014) Implications for multiple sclerosis in the era of the affordable care act: A clinical overview. Am J Manag Care 20: S220-227.

20. Howard J, Trevick S, Younger DS (2016) Epidemiology of multiple sclerosis. Neurol Clin 34: 919-939.

21. Mokry LE, Ross S, Timpson NJ, Sawcer S, Davey Smith G, et al. (2016)
Obesity and multiple sclerosis: A Mendelian randomization study. PLoS Med 13: e1002053.

22. Khare S, Seth D (2015) Lhermitte's sign: The current status. Ann Indian Acad Neurol 18: 154-156.

23. Rammohan KW (2009) Cerebrospinal fluid in multiple sclerosis. Ann Indian Acad Neurol 12: 246-253.

24. Bourre B, Zéphir H, Ongagna JC, Cordonnier C, Collongues N, et al. (2012) Longterm follow-up of acute partial transverse myelitis. Arch Neurol 69: 357-362.

25. Pandit $L$ (2009) Transverse myelitis spectrum disorders. Neurol India 57: 126133.

26. Pekcevik $\mathrm{Y}$, Mitchell $\mathrm{CH}$, Mealy MA, Orman $\mathrm{G}$, Lee $\mathrm{IH}$, et al. Differentiating neuromyelitis optica from other causes of longitudinally extensive transverse myelitis on spinal magnetic resonance imaging. Mult Scler 22: 302-311.

27. Wingerchuk DM, Lennon VA, Lucchinetti CF, Pittock SJ, Weinshenker BG (2007) The spectrum of neuromyelitis optica. Lancet Neurol 6: 805-815.

28. Lana-Peixoto MA, Callegaro D (2012) The expanded spectrum of neuromyelitis optica: Evidences for a new definition. Arq Neuropsiquiatr 70: 807-813.

29. Yokote H, Nose Y, Ishibashi S, Tanaka K, et al. (2015) Spinal cord ring enhancement in patients with neuromyelitis optica. Acta Neurol Scand 132: 37-41.

30. Kister I, Johnson E, Raz E, Babb J, Loh J et al. (2016) Specific MRI findings help distinguish acute transverse myelitis of neuromyelitis Optica from spinal cord infarction. Mult Scler Relat Disord 9: 62-67.

31. McKeon A (2013) Paraneoplastic and other autoimmune disorders of the central nervous system. Neurohospitalist 3: 53-64.

32. Å $\square$ ukjanowicz M, Brzosko M (2009) Myelitis in the course of systemic lupus erythematosus: Review. Pol Arch Med Wewn 119: 67-73.

33. Huang LK, Chung CC, Chen BZ, Chi NF, Hu CJ (2013) Systemic lupus erythematosus presented as extensive longitudinal myelitis. Acta Neurol Taiwan 22: 67-71.

34. Suzuki Y (2002) Transverse myelitis in systemic lupus erythematosus. Intern Med 41: 327-328.

35. Nardonea R, Fitzgeraldd RT, Baileye A, Zuccoli G (2015) Longitudinally extensive transverse myelitis in systemic lupus erythematosus: Case report and review of the literature. Clin Neurol Neurosurg 129: 57-61.

36. Figueroa D, Isache C, Sands M, Guzman N (2016) An unusual case of acute transverse myelitis caused by HSV-1 infection. IDCases 5: 29-31.

37. Eberhardt O, Küker W, Dichgans J, Weller M (2004) HSV-2 sacral radiculitis (Elsberg syndrome). Neurology 63: 758-759.

38. Amaral DM, Parreira T, Sampaio M (2015) Longitudinally extensive transverse myelitis and meningitis due to a rare infectious cause. BMJ Case Rep

39. .Chanthamat N, Sathirapanya $P(2010)$ Acute transverse myelitis associated with dengue viral infection. J Spinal Cord Med 33: 425-427.

40. Pappamikail L, Fernandes P, Gonçalves C (2014) Medullary schistosomiasis. Surg Neurol Int 5: 66.

41. VL Wolf, PJ Lupo, Lotze TE (2012) Pediatric acute transverse myelitis overview and differential diagnosis. J Child Neurol 27: 1426-1436.

42. Makhani N, Morris SK, Page AV, Brophy J, Lindsay LR, et al. (2011) A twist on Lyme: The challenge of diagnosing European Lyme neuroborreliosis. J Clin Microbiol 49: 455-457.

43. Schwenkenbecher P, Pul R, Wurster U, Conzen J, Pars K, et al (2017) Common and uncommon neurological manifestations of neuroborreliosis leading to hospitalization. BMC Infect Dis 17: 90.

44. Briani C, Dalla Torre C, Citton V, Manara R, Pompanin S, et al. (2013) Cobalamin deficiency: clinical picture and radiological findings. Nutrients 5: 4521-4539.

45. Jaiser SR, Winston GP (2010) Copper deficiency myelopathy. J Neurol 257 869-881.

46. Diaz RJ, Wong JH (2008) Spinal dural arteriovenous fistula: A treatable cause of myelopathy. CMAJ 178: 1286-1288.

47. Chen JY, Hsu HL, Huang YL, Chen CJ, Tseng YC, et al. (2015) Spinal dura arteriovenous fistula: Imaging features and its mimics. Korean J Radiol 16 $1119-1131$. 